Disponível em www.scielo.br/paideia

\title{
Escala de atitudes face ao lazer em adolescentes e jovens adultos
}

\author{
Teresa Freire \\ Universidade do Minho, Portugal \\ Carla Fonte \\ Universidade Fernando Pessoa, Portugal
}

\begin{abstract}
Resumo: Atualmente, o lazer e as atitudes face ao lazer têm sido alvo de interesse na investigação psicológica, concretamente no domínio da Psicologia Social. O objetivo do presente estudo foi adaptar a escala Leisure Attitude Scale de Raghb e Beard, construída para avaliar as atitudes face ao lazer em função das três componentes de atitude (cognitiva, afetiva e comportamental) para a população de estudantes, adolescentes e jovens adultos portugueses. Participaram no estudo 462 sujeitos (57,8\% do sexo feminino e $42,2 \%$ do sexo masculino), com uma média de idades de 19,97 (desvio padrão de 4,85). Os resultados obtidos confirmam a estrutura e as características psicométricas da versão original, refletindo valores largamente aceitáveis de fidelidade (consistência interna) e validade (validade de conteúdo) quer para a escala total quer para as várias sub-escalas (cognitiva, afetiva e comportamental). Conclui-se pela adequação da escala na medida das atitudes face ao lazer, no contexto português.
\end{abstract}

Palavras-chave: Lazer. Escala de atitudes. Adaptação. Medida. Fidelidade. Validade.

\section{Leisure attitude scale in adolescents and young adults}

\begin{abstract}
Nowadays, the leisure and its attitudes constitute an important issue in Social Psychology research. The aim of this study was to realize the adaptation of the Leisure Attitude Scale (Ragheb and Beard) for Portuguese individuals, including adolescents and young adults. This scale measures leisure attitude according to the three components (cognitive, affective and behavioural). A sample of 462 students (57.8\% girls and $42.2 \%$ boys) with a mean age of 19.97 (standard deviation of 4.85). The results confirm the original scale. Validity measures of internal consistency and content validity in the total scale and in the three subscales (Cognitive, affective and behavioural). The scale is an adequate instrument to measure leisure attitudes in the Portuguese context.
\end{abstract}

Keywords: Leisure. Attitude scale. Adaptation. Measurement. Reliability. Validity.

\section{Escala de actitud ante la diversión en adolescentes y jóvenes adultos}

Resumen: La diversión y las actitudes frente a ella vienes siendo objeto de interés en la investigación de la Psicología Social. Nuestro objetivo fue adaptar la escala Leisure Actitudes Scale de Raghb y Beard, que evalúa actitudes frente a la diversión en función de tres componentes (cognitivo, afectivo , y comportamental), para la población de estudiantes, adolescentes y jóvenes adultos portugueses. Participaron 462 sujetos (57,8\% del sexo femenino y $42,2 \%$ del sexo masculino), con media de edades de 19,97 (desviación standar de 4,85). Se confirmó la estructura y características psicométricas de la versión original, reflejando valores de fidelidad (consistencia interna) y validad (validad de contenido) en la escala total y en las varias sub-escalas (cognitiva, afectiva y comportamental). Concluimos que fue realizada la adaptación de la escala en el contexto portugués.

Palabras clave: Recreación. Escala de actitudes. Adaptación. Medida. Fidelidad. Validad. 
O estudo do lazer e seu impacto na vida cotidiana dos indivíduos tem merecido lugar de destaque na literatura psicológica, concretamente no domínio da Psicologia Social. Compreender de que forma as atividades de lazer estruturam e influenciam as experiências de vida, e ainda como os indivíduos se posicionam face às atividades e experiências de lazer ou que tipo de envolvimento têm, são alguns dos principais objetivos neste domínio de investigação.

Efetivamente um dos fatores determinantes do envolvimento em atividades de lazer são as atitudes face a ele. Uma revisão da literatura mostra que, em geral, atitudes positivas tendem a estar relacionadas com maior envolvimento em atividades de lazer, para além do que as experiências que daí decorrem tendem também a ser avaliadas de forma positiva (Freire, 2004; Ragheb \& Tate, 1993; Siegenthaler \& O'Dell, 2000). Neste sentido, a medida da atitude face ao lazer constitui um alvo privilegiado de estudo, não só por permitir um conhecimento maior acerca dos processos sócio-cognitivos envolvidos quando da realização de atividades de lazer, mas também porque possibilita a estruturação de formas de intervenção no sentido de promover nas pessoas atitudes mais positivas face ao lazer e com isso aumentar os níveis de bem-estar individual e social (Freire, 2001a, 2006; Haworth \& Lewis, 2005; Stebbins, 2000, 2006).

Apesar desta evidência conceitual e empírica, os estudos sobre atitudes face ao lazer mostram-se ainda escassos, pois também são poucos os instrumentos de medida diretamente relacionados com esta temática; existem alguns que avaliam diferentes dimensões relacionadas com as atividades ou experiências de lazer, sendo este entendido no seu sentido global, como a motivação para ele, a satisfação que produz, e outros mais (Barnett, 2005; Munchua-DeLisle \& Reddon, 2005; Witt \& Ellis, 1989), mas são poucos os que dizem diretamente respeito às atitudes face ao lazer. Neste âmbito, é possível referir-se às escalas de Burdge (1961), Neulinger e Breit (1971), Crandall e Slivken (1978, 1980), e de Ragheb e Beard (1982), todas dirigidas para o estudo das atitudes face ao lazer em geral. A principal diferença entre estas quatro escalas, para além de aspectos relativos ao conteúdo e número de itens, está ao nível do conceito de atitude, ao serem utilizados não apenas os seus três componentes na conceitualização e nas medidas feitas em relação à atitude geral do indivíduo.

Efetivamente, o conceito de atitude tem sofrido mudanças conceptuais ao longo do tempo, traduzindose em diferentes definições e operacionalizações do constructo, sustentados em diversos modelos e abordagens teóricas. Independentemente destes, e atendendo à multiplicidade de definições ainda hoje bem patentes na literatura, os autores são unânimes em considerar que este conceito é uma realidade psico-social ambígua e difícil de apreender (Albarracín, Johnson \& Zanna, 2005).

Vários modelos são apresentados na literatura psico-social, sendo alvo de atenção especial entre os investigadores os que conceitualizam e operacionalizam a atitude em torno de três componentes e que caracterizam os chamados de tripartidos. Dentre estes salienta-se, num primeiro momento, o modelo clássico, proposto por Rosenberg e Hovland (1960), e que permitiu desde logo a operacionalização dos três componentes de atitude: o cognitivo, que diz respeito às crenças, conhecimentos, informações e opiniões, sejam eles conscientes ou inconscientes, através das quais a atitude é expressa; o afetivo, que se refere aos sentimentos e às respostas fisiológicas expressas na atitude; e, finalmente, o comportamental que diz respeito aos processos que permitem a estruturação de uma intenção do comportamento e preparam o indivíduo para agir de determinada maneira. No entanto, este modelo não deixou claro de que forma cada um dos componentes contribuía para a atitude geral do indivíduo; este aspecto, posteriormente passou a ser alvo de estudos empíricos, na tentativa de testarem as contribuições independentes dos componentes cognitivo, afetivo e comportamental frente à atitude global (Breckler, 1984). É nesta linha de investigação que surge o modelo tripartido clássico revisto, criado por Zanna e Rempel (1988). Estes autores defendem a dimensão avaliativa da atitude, dando a cada um dos componentes uma contribuição independente na construção e expressão da atitude global. Este modelo abriu novas perspectivas de investigação, nomeadamente no nível da análise da discrepância entre medidas empíricas dos três componentes da mesma atitude e consequentemente da conceituação do que ela vem a ser. Neste sentido, 
a questão da relação entre atitude e comportamento, ou seja, perceber se elas predizem ou não a ele, são ainda atualmente tópicos relevantes e em aberto para pesquisa neste domínio científico (Neto, 1998).

Assim, se o conceito de atitude baseado nos três componentes dela - o cognitivo, o afetivo e o comportamental - é de particular relevância para o estudo das atitudes em geral, ele o é igualmente no estudo específico das atitudes face ao lazer já que contribui para a investigação de aspectos psico-sociais e sócio-cognitivos do lazer, em como conhecer e compreender o grau e tipo de envolvimento dos sujeitos em atividades de lazer, bem como as formas e processos que estão subjacentes à mudança de atitudes em relação ao lazer e qualidade de vida, ou ainda conhecer as atitudes face ao lazer em grupos ou contextos específicos (Hsieh, Spaulding, \& Riney, 2004; Lloyd \& Auld, 2002).

Atendendo às escalas existentes sobre atitudes face ao lazer, a de Ragheb e Beard (1982) surge como a única que mede os três componentes da atitude cognitivo, afetivo e comportamental - separadamente, tendo sido construída intencionalmente com esse objetivo, já que as anteriores não o faziam, e tendo-se que na perspectiva destes autores tratava-se de uma limitação considerável no estudo das atitudes face ao lazer. Assim, optou-se por trabalhar a escala Leisure Attitude Scale de Ragheb e Beard (1982) por se basear no modelo dos três componentes da atitude e ser largamente utilizada na investigação empírica atual. De acordo com a conceituação defendida por estes mesmos autores em relação às atitudes face ao lazer, o componente cognitivo diz respeito ao conhecimento e crenças gerais do indivíduo acerca do lazer, suas características e como se relacionam com a qualidade de vida de cada um. O componente afetivo, é relativo aos sentimentos do indivíduo face ao seu lazer, o grau em que gosta ou não das atividades e experiências de lazer. E, finalmente, o componente comportamental, é relativo às ações passadas, presentes e intenções do indivíduo frente às atividades e experiências de lazer.

O objetivo do presente estudo foi adaptar a referida escala para a população de jovens portugueses, integrando adolescentes e jovens adultos, visando contribuir para a existência de instrumentos de medida no domínio das atitudes face ao lazer.

\section{Método}

\section{Participantes}

Participaram no estudo 462 estudantes, adolescentes de 14 a 18 anos de idade, e jovens adultos de 19 a 39 anos de idade, oriundos de Escolas de Ensino Básico e de Universidades em Portugal. Destes, 267 eram do sexo feminino $(57,8 \%)$ e 195 do masculino $(42,2 \%)$. Em relação à idade dos participantes, a média foi de 19,97 (mínima de 14 e máxima de 39) e o desvio-padrão 4,85.

$\mathrm{Na}$ tabela 1 apresentam-se os dados da amostra em estudo, em função do grupo etário e sexo dos participantes.

Tabela 1. Caracterização da amostra: Sexo e Idade (média e desvio padrão)

\begin{tabular}{|l|c|c|c|c|}
\hline \multirow{2}{*}{} & \multicolumn{2}{|c|}{ Sexo } & \multicolumn{2}{c|}{ Idade } \\
\cline { 2 - 5 } & Feminino & Masculino & Média & $\begin{array}{c}\text { Desvio } \\
\text { padrão }\end{array}$ \\
\hline Adolescentes & 86 & 74 & 14.98 & 2.23 \\
\hline $\begin{array}{l}\text { Jovens } \\
\text { adultos }\end{array}$ & 181 & 121 & 22.61 & 3.63 \\
\hline Total & 267 & 195 & 19.97 & 4.85 \\
\hline
\end{tabular}

\section{Instrumentos}

Utilizou-se a escala original de Ragheb \& Beard (1982) - Leisure Attitude Scale; ela é composta por 36 itens, divididos em três sub-escalas relativas aos três componentes da atitude - cognitivo, afetivo e comportamental. Cada sub-escala é contem 12 itens, todos direcionados para o sentido positivo da atitude. $\mathrm{O}$ sistema de resposta utilizado é de tipo Likert, com cinco níveis de resposta referentes a acordo e desacordo, sendo que 1 revela uma atitude extrema desfavorável ou negativa ("Discordo totalmente") e 5 uma atitude extrema favorável ou positiva ("Concordo totalmente"). O ponto 3 corresponde a um nível neutro quanto à direção da atitude ("Nem discordo nem concordo"). Os valores das medidas da atitude, quer para a escala geral quer para cada uma das sub-escalas, são obtidos através da adição aritmética das respostas dadas pelo participante nos respectivos itens. Assim para cada sub-escala o valor total mínimo possível é 12 e o máximo é 60 (ponto neutro situado no valor 36). Já em relação à escala total o valor mínimo possível é 
36 e o máximo 180 (ponto neutro situado no valor 108). Valores mais elevados (acima do ponto neutro) revelam atitudes mais positivas e, pelo contrário, os mais baixos (abaixo do ponto neutro) indicam atitudes mais negativas em relação ao lazer.

\section{Procedimento}

Num primeiro momento a escala original de Ragheb e Beard (1982) foi traduzida para o português, mantendo-se exatamente a mesma estrutura em termos do número de itens e sub-escalas, e as alternativas de resposta (escala tipo Likert de cinco pontos), o que teve como base, após uma análise, o fato de se considerar que eram eficazes para se fazer a avaliação e a medida (Almeida \& Freire, 2003; Freire \& Almeida, 2001b). A versão portuguesa da escala ficou com o título de "Escala de Atitudes face ao Lazer" (Freire \& Fonte, 2006).

Num segundo momento, as escalas foram administradas aos participantes nas respectivas instituições de ensino, fora do contexto de sala de aula. A seleção deles foi feita aleatoriamente e todos que participaram do estudo o fizeram voluntariamente. Os participantes responderam às perguntas do questionário logo após ele ter sido entregue, devolvendo-o de imediato, tendo-se como tempo de resposta associado à escala o mínimo de 10 e o máximo de 15 minutos, aproximadamente.

\section{Resultados}

As etapas realizadas para a análise das qualidades psicométricas da escala portuguesa seguiram os mesmos passos utilizados por Ragheb e Beard (1982) na construção da sua escala original, consistindo nos seguintes procedimentos: análise descritiva e índice de fidelidade (valores de média e desvio padrão; alpha de Cronbach); estrutura fatorial (análise fatorial de componentes principais); e correlações entre sub-escalas (correlações de Pearson).

\section{Estatística descritiva e índice de fidelidade}

Tal como o obtido na escala original de 1982 , nesta versão portuguesa, a média de respostas para os três componentes reflete, em termos globais, uma atitude positiva dos participantes em relação às atividades de lazer. Nesta versão portuguesa, o componente cognitivo é o que obtém a média mais elevada, e o comportamental a menos elevada, resultados estes diferentes dos obtidos por Ragheb e
Beard em 1982, em que a média mais elevada era no componente afetivo e a menor na escala cognitiva. Os resultados acham-se na Tabela 2.

Tabela 2. Estatísticas descritivas e valores de fidelidade para a Escala de Atitudes face ao Lazer (Freire \& Fonte, 2006)

\begin{tabular}{|l|c|c|c|c|c|}
\hline $\begin{array}{c}\text { Componentes e } \\
\text { escala total }\end{array}$ & $\begin{array}{c}\text { Número } \\
\text { de itens }\end{array}$ & Média & $\begin{array}{c}\text { Desvio } \\
\text { Padrão }\end{array}$ & $\begin{array}{c}\text { Min- } \\
\text { Máx }\end{array}$ & $\begin{array}{c}\text { Alpha de } \\
\text { Cronbach }\end{array}$ \\
\hline Cognitivo & 12 & 50.64 & 5.67 & $29-60$ & 0.86 \\
\hline Afetivo & 12 & 48.86 & 5.70 & $24-60$ & 0.86 \\
\hline Comportamental & 12 & 43.96 & 6.09 & $24-60$ & 0.79 \\
\hline Total & 36 & 143.40 & 14.77 & $85-180$ & 0.91 \\
\hline
\end{tabular}

No entanto, e apesar de os valores médios obtidos traduzirem atitudes positivas, os mínimos revelam a existência de respostas que traduzem atitudes negativas, em qualquer uma das sub- escalas e na escala total, embora o valor mínimo possível nunca tenha sido atingido. Estes resultados são relevantes já que demonstram que a escala mostrase capaz de possibilitar o discriminar entre atitudes negativas e positivas face ao lazer.

Os valores de fidelidade alpha de cada uma das sub-escalas e da escala total, refletem valores largamente aceitáveis entre 0,79 e 0,91 (Freire \& Almeida, 2001b; Loewenthal, 2001), tal como na original. Em ambas as escalas (original e portuguesa), o valor de alpha mais elevado é obtido para a total e o menor para a sub-escala comportamental.

Concretamente em relação às sub-escalas, o valor mais elevado de fidelidade foi obtido em ambas as sub-escalas - cognitiva e afetiva - contrariamente à versão original em que este valor aparece na subescala afetiva. Refere-se que no presente estudo os valores de alpha obtidos para cada componente são os mais elevados para esse conjunto de itens, não havendo nenhum item que, se retirado, aumente o valor de fidelidade em causa.

Estes resultados obtidos com o índice de fidelidade alpha de Cronbach traduzem a consistência interna associada aos itens que compõem a versão portuguesa da escala, ou seja, existe um elevado grau de uniformidade e coerência nas respostas dos 
Tabela 3. Estrutura fatorial da Escala de atitudes face ao lazer (Freire \& Fonte, 2006)

\begin{tabular}{|c|c|c|c|}
\hline ITEM & $\begin{array}{c}\text { FACTOR } \\
\text { I }\end{array}$ & $\begin{array}{c}\text { FACTOR } \\
\text { II }\end{array}$ & $\begin{array}{c}\text { FACTOR } \\
\text { III }\end{array}$ \\
\hline 1 - Envolver-se em atividades de lazer é uma boa forma de usar o tempo & & 0,610 & \\
\hline 2 - As atividades de lazer são benéficas para os indivíduos e para as sociedades & & 0,689 & \\
\hline 3 - Normalmente as pessoas criam amizades nas suas atividades de lazer & & 0,476 & \\
\hline $4-$ As atividades de lazer contribuem para a saúde das pessoas & & 0,627 & \\
\hline 5 - As atividades de lazer aumentam a felicidade das pessoas & & 0,614 & \\
\hline 6 - O lazer aumenta a produtividade das pessoas no trabalho & & 0,550 & \\
\hline 7 -As atividades de lazer ajudam a renovar a energia de uma pessoa & & 0,624 & \\
\hline 8 - As atividades de lazer podem ser uma forma de auto-crescimento & & 0,591 & \\
\hline 9 - As atividades de lazer ajudam os indivíduos a relaxar & & 0,567 & \\
\hline $10-$ As pessoas precisam de atividades de lazer & & 0,450 & \\
\hline 11 - As atividades de lazer são boas oportunidades para contactos sociais & & 0,595 & \\
\hline 12 - As atividades de lazer são importantes & & 0,477 & \\
\hline \multicolumn{4}{|l|}{ Itens relativos à componente cognitiva (12 itens) } \\
\hline 13 - Quando estou envolvido(a) em atividades de lazer o tempo voa & 0,582 & & \\
\hline 14 - As minhas atividades de lazer dão-me prazer & 0,662 & & \\
\hline 15 - Eu valorizo as minhas atividades de lazer & 0,595 & & \\
\hline 16 - Durante as minhas atividades de lazer posso ser eu próprio & 0,469 & & \\
\hline 17 - As minhas atividades de lazer propiciam-me experiências encantadoras & 0,541 & & \\
\hline 18 - Sinto que o lazer é bom para mim & 0,626 & & \\
\hline 19 - Gosto do tempo que passo quando estou envolvido em atividades de lazer & 0,67 & & \\
\hline 20 - As minhas atividades de lazer são reparadoras & 0,55 & & \\
\hline 21 - Considero apropriado um envolvimento frequente em atividades de lazer & 0,415 & & \\
\hline 22 - Sinto que o tempo que passo em lazer não é tempo perdido & 0,543 & & \\
\hline 23 - Gosto das minhas atividades de lazer & 0,703 & & \\
\hline 24 - As minhas atividades de lazer absorvem-me ou exigem a minha total atenção & 0,43 & & \\
\hline \multicolumn{4}{|l|}{ Itens relativos à componente afetiva (12 itens) } \\
\hline $25-$ Faço atividades de lazer frequentemente $*$ & 0,563 & & 0,308 \\
\hline $\begin{array}{l}26 \text { - Se tivesse oportunidade aumentaria a quantidade de tempo que passo em } \\
\text { atividades de lazer }\end{array}$ & & & 0,388 \\
\hline $\begin{array}{l}27 \text { - Compro artigos e equipamentos para usar nas minhas atividades de lazer sempre } \\
\text { que as minhas possibilidades financeiras assim o permitem }\end{array}$ & & & 0,417 \\
\hline 28 - Faria mais e novas atividades de lazer se tivesse mais tempo e dinheiro & & & 0,362 \\
\hline $\begin{array}{l}29 \text { - Dedico bastante tempo e esforço para me tornar mais competente nas minhas } \\
\text { atividades de lazer }\end{array}$ & & & 0,54 \\
\hline $\begin{array}{l}30 \text { - Se pudesse escolher, viveria num ambiente ou cidade que tivesse mais } \\
\text { oportunidades de lazer }\end{array}$ & & & 0,455 \\
\hline 31 - Faço algumas atividades de lazer, mesmo quando elas não foram planejadas & & & 0,417 \\
\hline $\begin{array}{l}32 \text { - Participaria numa aula ou seminário para ser mais capaz de fazer melhor as } \\
\text { atividades de lazer }\end{array}$ & & & 0,565 \\
\hline $\begin{array}{l}33 \text { - Apoio a ideia de aumentar o meu tempo livre para poder me envolver em mais } \\
\text { atividades de lazer }\end{array}$ & & & 0,498 \\
\hline 34 - Envolvo-me em atividades de lazer mesmo quando ando ocupado & & & 0,537 \\
\hline 35 - Gostaria de ter mais tempo de educação e preparação para atividades de lazer. & & & 0,625 \\
\hline 36 - Dentre as atividades que tenho dou grande prioridade às de lazer. & 0,288 & & 0,582 \\
\hline \multicolumn{4}{|l|}{ Itens relativos ao componente comportamental (12 itens) } \\
\hline \multirow{2}{*}{ Variância explicada (Escala Total) $=39 \%$} & Factor I & Factor II & Factor III \\
\hline & $15,91 \%$ & $13,97 \%$ & $9,12 \%$ \\
\hline
\end{tabular}

* Único item que se correlaciona mais com o fator que não é o esperado em termos de componente de atitude, $\mathrm{d} \epsilon$ acordo com a versão original 
participantes a cada um dos itens que compõem a escala total e respectivas sub-escalas (Almeida \& Freire, 2003).

\section{Estrutura Fatorial}

Os dados obtidos com a administração da escala aos 462 participantes foram analisados em relação à sua estrutura fatorial usando a Análise Fatorial dos Componentes Principais. Tal como realizado na da versão original (1982), três fatores foram rodados através da solução Varimax, sendo os resultados da matriz apresentados na Tabela 3.

A estrutura fatorial encontrada confirma a diferenciação dos três componentes de atitude tal como conceitualmente definidas por Ragheb e Beard (1982). Ou seja, de acordo com os resultados, os itens que saturam cada um dos fatores correspondem aos definidos para os componentes de atitude (cognitivo, afetivo e comportamental). Assim o fator I (porcentagem mais elevada de variância explicada) integra todos os itens relativos ao componente afetivo ; o fator II integra todos os relativos ao componente cognitivo e o fator III, todos os que são relativos ao componente comportamental (porcentagem menos elevada de variância explicada)

Na versão original, os autores tomaram como critério de inclusão de um item num fator, a sua maior correlação com um fator, comparativamente a qualquer um dos outros. Na versão portuguesa, tal aconteceu igualmente para a totalidade dos itens das várias componentes com excepção do item 25 ("Faço atividades de lazer frequentemente"). Embora este item tenha um valor de correlação aceitável com o fator correspondente à componente comportamental, tal como seria esperado atendendo à escala original, apresenta um valor mais elevado de correlação com o fator que nesta estrutura fatorial integra os itens da componente afetiva (Tabela 3 ).

No entanto, e apesar deste item saturar mais o fator relativo ao componente afetivo, é um contributo essencial para o valor de consistência interna associada aos que compõem o componente comportamental (valor de alpha de Cronbach apresentado na tabela 3 ), tal como definido na versão original de 1982. Ou seja, como já referido anteriormente, a retirada deste item da sub-escala não aumenta o valor de alpha obtido. De acordo com estes resultados optou-se por manter o item 25 na sub-escala relativa ao componente comportamental de acordo com a conceitualização subjacente à escala original, já que do ponto de vista psicométrico não existe qualquer prejuízo do valor obtido para o índice de fidelidade desta ou mesmo da escala total, e em termos da estrutura fatorial o valor de correlação deste item com o fator III é também aceitável.

$\mathrm{Na}$ sequência destes resultados, procedeu-se ainda à realização de uma análise fatorial com os valores obtidos para as três sub-escalas, com rotação varimax, e sem definição prévia do número de fatores a analisar. Os resultados mostram a formação de um só fator que inclui as três sub-escalas, sendo que a sub-escala cognitiva se correlaciona com of fator com um valor de 0,716, a afetiva com 0,793 e a comportamental com 0,651 . A variância total explicada por este único fator é de 71,97\%. Estes resultados permitem afirmar que as três sub-escalas estão efetivamente a medir diferentes aspectos de um mesmo traço ou construto (atitude face ao lazer), o que reforça a adequação destas três componentes na medida das atitudes face ao lazer. Ou seja, a existência de um só fator é a demonstração da construção teórica que está subjacente à própria escala (Ellis \& Witt, 1994).

Assim, e tal como na escala original (1982), a estrutura fatorial confirma a existência de validade de conteúdo, ou seja, existe um elevado grau de adequação dos itens à dimensão do comportamento avaliada pela escala total e, também, pelas respectivas sub-escalas (Almeida \& Freire, 2003).

\section{Correlações entre sub-escalas}

As correlações obtidas entre as sub-escalas cognitiva, afetiva e comportamental são apresentadas na Tabela 4.

Tabela 4. Correlações entre as subescalas e a escala total

\begin{tabular}{|l|c|c|c|}
\hline & Afetiva & Comportamental & $\begin{array}{c}\text { Escala } \\
\text { Total }\end{array}$ \\
\hline Cognitiva & $0,660^{* *}$ & $0,491^{* *}$ & $0,838^{* *}$ \\
\hline Afetiva & 1 & $0,587^{* *}$ & $0,880^{* *}$ \\
\hline Comportamental & & 1 & $0,825^{* *}$ \\
\hline
\end{tabular}

** correlação de Pearson, $\mathrm{p}<0,01$

Verifica-se que os valores de correlação variam entre 0,49 e 0,66 (entre sub-escalas) ou entre 0,83 e 
0,88 quando se entra com as correlações com a escala total (na versão original todos estes valores variaram entre 0,47 e 0,63 entre sub-escalas e entre 0,68 e 0,82 , relacionando com a escala total).

Tal como na escala original a correlação entre os resultados da sub-escala afetiva e comportamental $(\mathrm{r}=0,59 ; \mathrm{p}<0,01)$ é maior do que a obtida nas cognitiva e comportamental $(r=0,49 ; \mathrm{p}<0,01)$. Ragheb e Beard (1982) salientam este tipo de resultados discutindo possíveis causas das intenções comportamentais em termos dos componentes das atitudes. Neste sentido colocam a hipótese das intenções comportamentais poderem ser causadas mais pelo que é sentido do que pelo que é sabido acerca das atividades de lazer. Algo que apesar de se observar também nos resultados com os participantes portugueses, fica em aberto criando novas pistas para estudos futuros, sobretudo no âmbito da relação entre atitude e comportamento face ao lazer, bem como em relação à análise das contribuições que cada um dos componentes tem na atitude manifesta pelo participante.

\section{Considerações Finais}

A versão portuguesa adaptada da escala de Ragheb e Beard (1982), apresenta-se como um instrumento com características psicométricas adequadas em termos de validade e fidelidade tornando possível a avaliação das atitudes face ao lazer em adolescentes e jovens adultos no contexto português.

A validação da escala na sua versão portuguesa mantendo as características psicométricas da versão original, reforça a conceituação subjacente à construção da escala original permitindo a medida das atitudes face ao lazer de acordo com os componentes cognitivo, afetivo e comportamental, em contextos culturais diferentes. Neste sentido, trata-se de uma escala que contribui para uma análise consistente dos processos sócio-cognitivos associados às atitudes face ao lazer.

Salienta-se, então, a obtenção de atitudes positivas face ao lazer (independentemente dos componentes em avaliação), o que confirma os resultados presentes na literatura no sentido de os indivíduos apresentarem, em geral, uma percepção positiva associada às atividades e experiências de lazer. Este resultado sublinha a importância da positividade associada às atividades e experiências de lazer e suas implicações quer ao nível do envolvimento dos indivíduos quer da sua utilização para fins desenvolvimentais e de promoção de competências.

Os diferentes resultados obtidos, em função das atitudes expressas através das várias sub-escalas e da escala total reforçam a importância do estudo das atitudes sustentado pelo modelo dos três componentes, e das suas implicações na investigação quanto à relação entre atitudes e comportamentos. Concretamente no domínio do lazer, estes aspectos traduzem-se não só no nível do conhecimento dos processos sócio-cognitivos como também no da intervenção, na definição de estratégias de envolvimento em atividades de lazer e adesão a oportunidades estruturadas e oferecidas pela comunidade. Ou seja, as implicações sociais associadas ao estudo das atitudes face ao lazer adquirem também relevância neste domínio de investigação. Neste âmbito, destacam-se no estudo efetuado, os valores mais elevados no componente cognitivo da atitude e os menos elevados no componente comportamental. Este aspecto pode denotar alguma discrepância entre as crenças e conhecimentos acerca do lazer e do seu impacto positivo na vida dos adolescentes e jovens adultos portugueses e a sua efetiva concretização em atividades e comportamentos de lazer na vida diária. Ou seja, se os participantes deste estudo demonstram uma atitude bastante favorável em termos de conhecimentos e crenças associados ao lazer, o mesmo não acontece no nível dos comportamentos passados, presentes ou das intenções comportamentais em relação a ações e atividades relacionadas com o lazer. Tais resultados mantêm em aberto a discussão em torno dos modelos tripartidos no estudo das atitudes.

Em síntese, a escala aqui apresentada constitui um instrumento válido no estudo da medida das atitudes face ao lazer e um contributo para a investigação no domínio dos processos sóciocognitivos relacionados com o lazer e seu impacto na vida dos indivíduos, concretamente no contexto português. Como tal, a realização deste tipo de estudos em outros contextos culturais e/ou com 
grupos associados a populações específicas deve se constituir em um objetivo fundamental, dando continuidade neste domínio da investigação das atitudes e lazer.

\section{Referências}

Albarracín, D., Johnson, B. T., \& Zanna, M. P. (2005). The handbook of attitudes. Mahwah, NJ: Erlbaum.

Almeida, L., \& Freire, T. (2003). Metodologia de Investigação em Psicologia e Educação. Braga: Psiquilíbrios.

Barnett, L.A. (2005). Measuring the ABCs of leisure experience: awareness, boredom, challenge, distress. Leisure Sciences, 27(2), 131-155.

Breckler, S. (1984). Empirical validation of affect, behavior, and cognition as distinct components of attitude. Journal of Personality and Social Psychology, 47, 1191-1205.

Burdge, R. (1961). The development of a leisureorientation scale. Columbus, Ohio: Ohio State University.

Crandall, R., \& Slivken, K. (1978). The importance of measuring leisure attitudes. In Symposium, National Recreation and Park Association, 1978, Miami Beach, Florida.

Crandall, R., Slivken, K. (1980), Leisure attitudes and their measurement. In Iso-Ahola, S. (Eds), Social Psychological Perspectives on Leisure and Recreation, C.C. Thomas, Springfield, il., pp.261-84.

Ellis, G. D., \& Witt, P. A. (1994). Perceived freedom in leisure and satisfaction: Exploring the fator structure of the perceived freedom components of the leisure diagnostic battery. Leisure Sciences, 16(4), 259-270.

Freire, T. (2001a). Ócio e tempo livre: perspectivar o lazer para o desenvolvimento. Revista GalegoPortuguesa de Psicologia e Educación, 7(5), 345-349.

Freire, T. (2004). Psicologia social do lazer. Em F. Neto (Org.), Psicologia social aplicada. Lisboa: Universidade Aberta.
Freire, T. (2006). Leisure experience and positive development of adolescents: from theory to intervention. In A. Delle Fave (Ed.), Dimensions of well-being. Research and intervention (pp. 366-381). Milano: Franco Angeli.

Freire, T., \& Almeida, L. (2001b). Escalas de avaliação: Construção e validação. Em M. Fernandes \& L.S.Almeida (Orgs.), Métodos e técnicas de avaliação: Contributos para a prática e investigação psicológicas (pp.109128). Braga: Universidade do Minho.

Freire, T. \& Fonte, C. (2006). Escala de Atitudes face ao Lazer. Tradução e adaptação de M. G. Ragheb \& J. G. Beard, 1982 - Versão para investigação. Universidade do Minho.

Haworth, J., \& Lewis, S. (2005). Work, leisure and well-being. British Journal of Guidance \& Counseling, 33 (1), 67-79.

Hsieh, S., Spaulding, A., \& Riney, M. (2004). A qualitative look at leisure benefits for Taiwanese nursing students. The Qualitative Report, 9(4), 604-629.

Lloyd, K. M., \& Auld, C. J. (2002). The role of leisure in determining quality of life: issues of content and measurement. Social Indicators Research, 57(1), 43-71.

Loewenthal, K. M. (2001). An introduction to psychological tests and scales. Cornwall: Psychology Press.

Munchua-DeLisle, M. M., \& Reddon, J. R. (2005). Leisure motivation in relation to psychosocial adjustment. Advances in Psychology Research, 33, 203-217.

Neulinger, J., \& Breit, M. (1971). A study of leisure. In J. Neulinger (Ed.), The psychology of leisure (pp.167-183). Springfield, III: Charles C. Thomas.

Neto, F. (1998). Psicologia social. Lisboa: Universidade Aberta.

Ragheb, M. G., \& Beard, J. G. (1982). Measuring Leisure Attitude. Journal of leisure Research, 14(2), 155-167. 
Ragheb, M. G., \& Tate, R. L. (1993). A behavioural model of leisure participation, based on leisure attitude, motivation and satisfaction. Leisure Studies, 12(1), 61-70.

Rosenberg, M. J., \& Hovland, C. I. (1960). Cognitive, affective and behavioral components of attitudes. In M. J. Rosemberg (Ed.), Attitude organization and change(pp. 1-14). New Haven, CT: Yale University Press.

Siegenthaler, K. L., \& O’Dell, I. (2000). Leisure attitude, leisure satisafaction, and perceived freedom in leisure within family dyads. Leisure Sciences, 22(4), 281-296.

Stebbins, R. A. (2000). Optimal leisure lifestyle: Combining serious and casual leisure for personal well-being. In M. C. Cabeza (Ed.), Leisure and human development: Proposals for the 6th World Leisure Congress (pp. 101-107). Bilbao, Spain: University of Deusto.

Stebbins, R. A. (2006). Serious leisure: A perspective for our time. New Brunswick, NJ: Aldine/Transaction.

Witt, P. A., \& Ellis, G. D. (1989). The Leisure Diagnostic Battery User's Manual. State College, Pennsylvania: Venture Publishing.

Zanna, M. P., \& Rempel, J. K. (1988). Attitudes: A new look to an old concept. In D. Bar-Tal, \& A. W. Kruglanski (Eds.), Social psychology of knowledge (pp.315-334). New York: Cambridge University Press.

Endereço para correspondência:

Teresa Freire. Instituto de Educação e Psicologia. Universidade do Minho, Campus de Gualtar, 4710-057 Braga, Portugal. Email: tfreire@iep.uminho.pt.

Artigo recebido em 19/12/2006.

Aceito para publicação em 06/04/2007. 